\title{
Feasibility of Salvage Re-irradiation With Stereotactic Radiotherapy for Recurrent Glioma Using CyberKnife
}

\author{
KANA ADACHI ${ }^{1}, \mathrm{KAZUHIKO} \mathrm{HAYASHI}^{2,3}$, NAOKI KAGAWA ${ }^{4}$, MANABU KINOSHITA ${ }^{4}$, \\ IORI SUMIDA ${ }^{2}$, YUICHI AKINO ${ }^{2}$, HIROYA SHIOMI ${ }^{2}$, KEISUKE TAMARI ${ }^{2}$, OSAMU SUZUKI ${ }^{2}$, \\ RYUICHI HIRAYAMA ${ }^{4}$, NORIYUKI KIJIMA ${ }^{4}$, FUMIAKI ISOHASHI ${ }^{2}$, YUJI SEO ${ }^{2}$, \\ KEISUKE OTANI $^{2}$, HARUHIKO KISHIMA ${ }^{4}$ and KAZUHIKO OGAWA ${ }^{2}$ \\ ${ }^{1}$ Department of Radiation Oncology, Toyonaka Municipal Hospital, Toyonaka, Japan; \\ ${ }^{2}$ Department of Radiation Oncology, Osaka University Graduate School of Medicine, Suita, Japan; \\ ${ }^{3}$ Osaka Heavy Ion Therapy Center, Osaka, Japan; \\ ${ }^{4}$ Department of Neurosurgery, Osaka University Graduate School of Medicine, Suita, Japan
}

\begin{abstract}
Aim: To evaluate the toxicity and efficacy of reirradiation with salvage stereotactic radiotherapy (SRT) for recurrent glioma using CyberKnife. Patients and Methods: This study retrospectively investigated 35 patients with 48 recurrent grade 2-4 gliomas who received SRT between 1998 and 2011. Six patients (17.1\%) had grade 2 gliomas, nine (25.7\%) had grade 3 gliomas, and 20 (57.1\%) had glioblastomas; all initially underwent surgery and conventional radiotherapy. The median initial and subsequent radiotherapy doses were 60 and $26 \mathrm{~Gy}$, respectively. Results: After a median follow-up period of 9.0 months, the only toxicity of grade 2 or more was radiationinduced brain necrosis in four patients (11.4\%). The median overall and progression-free survival periods following reirradiation were 9.0 and 3.0 months, respectively. Univariate analysis revealed that performance status at salvage reirradiation was a significant predictor of progression-free survival. Conclusion: Salvage re-irradiation using CyberKnife is feasible, with an acceptable toxicity profile, for patients with recurrent glioma.
\end{abstract}

Gliomas are relatively rare tumours, their annual incidence is approximately seven patients per 100,000 population (1). They are classified by cell type and grade according to the World Health Organization (WHO) classification of CNS

Correspondence to: Kazuhiko Hayashi, Kazuhiko Ogawa, Department of Radiation Oncology, Osaka University Graduate School of Medicine, 2-2 Yamadaoka, Suita City, Osaka 565-0871, Japan. Tel: +81668793482, Fax: +81668793489, e-mail: k.hayashi@osakahimak.or.jp

Key Words: Stereotactic radiotherapy, recurrent glioma, CyberKnife, re-irradiation, safety profile. tumours (2). Eighty-five percent of all diagnosed gliomas are high-grade (1), and their treatment strategies depend on the tumour's grade, location, and histological type. The standard initial treatment for high-grade glioma includes maximal surgical resection and postoperative chemoradiotherapy. However, intracranial recurrence frequently occurs despite multimodality treatments, with most recurrences occurring locally (typically within $2 \mathrm{~cm}$ of the primary lesion) (3).

A standard treatment regimen for gliomas that recur has yet to be established; treatment options are re-resection, chemotherapy (including with temozolomide or bevacizumab), and salvage radiotherapy (4). Patients with recurrent gliomas who are under 70 years of age, have tumour volumes $<50$ $\mathrm{cm}^{3}$, and have preoperative Karnofsky performance status scores $>80 \%$ are generally considered candidates for repeat resection (4); however, this treatment is often limited because of the infiltrative nature of gliomas. Alternatively, focal lesion recurrences may be treatable with salvage radiotherapy; however, patients who previously received intracranial radiotherapy at doses $>40$ Gy are at a high risk of radiationinduced toxicity, especially brain radionecrosis, upon reirradiation.

To date, many retrospective studies have found that stereotactic surgery (SRS) and stereotactic radiotherapy (SRT) employed via various methods are well-tolerated and effective (5-12). However, most of these studies were performed using a linear accelerator (Linac) or Gamma Knife (Elekta AB, Stockholm, Sweden). CyberKnife (Accuray Inc., Sunnyvale, CA, USA) combines computercontrolled robotics with real-time imaging guidance to provide high conformality without resorting to aggressive target fixation, thereby offering an attractive SRS or SRT option $(13,14)$. To our knowledge, only a few studies of reirradiation for recurrent gliomas using CyberKnife have been published $(11,12,14,15)$. Therefore, we performed this 
study to retrospectively evaluate the toxicity and efficacy of salvage re-irradiation with SRT for recurrent gliomas using CyberKnife.

\section{Patients and Methods}

This study was approved by the Institutional Review Board of Osaka University Hospital (No. 18512). Research was conducted in accordance with the Helsinki Declaration. Re-irradiation was defined as treatment in which the initial and post-recurrence radiotherapy planning target volumes (PTVs) overlapped.

Patients. A retrospective survey was carried out of all patients who were treated with salvage re-irradiation using CyberKnife for recurrent glioma after initial treatment at Osaka University Hospital. Thirty-five patients with 48 lesions who were treated between 1998 and 2011 were applicable. All patients were initially treated with surgery and conventional radiotherapy. The median dose of initial irradiation was $60 \mathrm{~Gy}$ (range $=40-80 \mathrm{~Gy}$ ). Recurrent gliomas were diagnosed by the presentation of new or increasingly contrastenhanced lesions on magnetic resonance imaging (MRI). Gliomas were graded according to the WHO classification of CNS tumours at the initial surgery (2).

CyberKnife treatment planning and delivery. Patients were immobilized with a custom thermoplastic head mask system and underwent a contrast-enhanced computed tomographic (CT) simulation in the treatment position with $1.0 \mathrm{~mm}$ slices; they also underwent brain MRIs to which the simulated CTs were fused. The gross tumour volume (GTV) was defined as the contrast enhancing CT-detectable lesion(s) and T1-weighted MRI images. The PTV was defined as the GTV plus a $1 \mathrm{~mm}$ margin to account for positioning errors. All treatment planning was performed using the CyberKnife Planning System (On Target-TPS; Accuray Inc., Sunnyvale, CA, USA). An inverse planning method with a non-isocentric technique was used. Total doses were prescribed to the isodose line that covered $90 \%$ of the PTV. The prescribed doses for re-irradiation were 9-30 Gy in 1 fraction for 29 lesions, 24-36 Gy in 3 fractions for 11 lesions, and 20-35 Gy in 5 fractions for eight lesions. The median doses of re-irradiation per fraction were 27 Gy for 1 fraction, $30 \mathrm{~Gy}$ for 3 fractions, and $25 \mathrm{~Gy}$ for 5 fractions. The most commonly prescribed dose was $30 \mathrm{~Gy}$ in 1 fraction (eight lesions), followed by $27 \mathrm{~Gy}$ in 1 fraction (seven lesions), 25 Gy in 5 fractions (five lesions), and $24 \mathrm{~Gy}$ in 1 fraction (five lesions). Total dose and fractionation were determined in consideration of the tumour size, dose of previous irradiation, proximity of organs-at-risk, and the patient's condition. All treatments were delivered with $6 \mathrm{MV}$ photons utilizing CyberKnife (G2) between 1998 and 2003 and CyberKnife II (G3) between 2004 and 2011.

Follow-up. After treatment, follow-up MRIs were performed every 3 months in the absence of any neurological deterioration. Toxicities were graded according to the National Cancer Institute's Common Terminology Criteria for Adverse Events (version 4.0) (16); we collected information on toxicities of grade 2 or more. The patients' performance statuses were scored according to the Eastern Cooperative Oncology Group criteria.

Statistical analyses. Progression-free survival (PFS) and overall survival (OS) were calculated using the Kaplan-Meier method. PFS
Table I. Patient and tumour characteristics $(N=35)$.

\begin{tabular}{|c|c|}
\hline Factor & Value \\
\hline \multicolumn{2}{|l|}{ Gender, N (\%) } \\
\hline Male & $24(68.6)$ \\
\hline Female & $11(31.4)$ \\
\hline \multicolumn{2}{|l|}{ Age at initial irradiation } \\
\hline Median, years (range) & $57(11-78)$ \\
\hline \multicolumn{2}{|c|}{ Glioma grades (WHO classification), $\mathrm{N}(\%)$} \\
\hline 2 & $6(17.1)$ \\
\hline 3 & $9(25.7)$ \\
\hline 4 & $20(57.1)$ \\
\hline \multicolumn{2}{|c|}{ Initial irradiation dose (Gy $[\mathrm{RBE}])$} \\
\hline Median (range) & $60(40-80)$ \\
\hline \multicolumn{2}{|c|}{ Interval between initial irradiation and } \\
\hline Median (range) & $11(1-126)$ \\
\hline \multicolumn{2}{|c|}{ Site of failure at salvage re-irradiation, $\mathrm{N}(\%)$} \\
\hline In-field & $32(91.4)$ \\
\hline Marginal & $2(5.7)$ \\
\hline Out of field & $1(2.9)$ \\
\hline \multicolumn{2}{|c|}{ Age at salvage re-irradiation (years) } \\
\hline Median (range) & $58(12-79)$ \\
\hline \multicolumn{2}{|c|}{ ECOG PS at salvage re-irradiation, $\mathrm{N}(\%)$} \\
\hline 0 & $10(28.6)$ \\
\hline 1 & $13(37.1)$ \\
\hline 2 & $3(8.6)$ \\
\hline 3 & $7(20.0)$ \\
\hline 4 & $2(5.7)$ \\
\hline \multicolumn{2}{|c|}{ Follow-up from salvage re-irradiation, months } \\
\hline Median (range) & $9(2-109)$ \\
\hline \multicolumn{2}{|c|}{ Salvage surgery before salvage re-irradiation } \\
\hline Yes & $3(8.6)$ \\
\hline No & $32(91.4)$ \\
\hline \multicolumn{2}{|c|}{ Chemotherapy at salvage treatment } \\
\hline Interferon-based & $20(57.1)$ \\
\hline Temozolomide-based & $6(17.1)$ \\
\hline Other & $5(14.3)$ \\
\hline \multicolumn{2}{|c|}{ Re-irradiation dose [Gy (RBE)] } \\
\hline Median (range) & $26(9-36)$ \\
\hline \multicolumn{2}{|c|}{ PTV at salvage re-irradiation, $(\mathrm{ml})$} \\
\hline Median (range) & $4.2(0.2-49.6)$ \\
\hline
\end{tabular}

RBE: Relative biological effectiveness; WHO: World Health Organization; ECOG PS: Eastern Cooperative Oncology Group performance status; PTV: planning target volume.

was defined as the interval between the date of commencing reirradiation and that of disease progression at any site, death from any cause, or the last follow-up. OS was defined as the interval between commencing re-irradiation and either death from any cause or the last follow-up date.

Univariate analyses of factors that predict PFS and OS were performed using the log-rank test. The patients were divided into subgroups according to the median values of age, interval between initial irradiation and re-irradiation, re-irradiation dose, and the PTV at re-irradiation. A two-tailed value of $p<0.05$ was considered statistically significant. All statistical analyses were conducted using the JMP statistical software (version 14.0; SAS Institute Inc., Cary, NC, USA). 
A

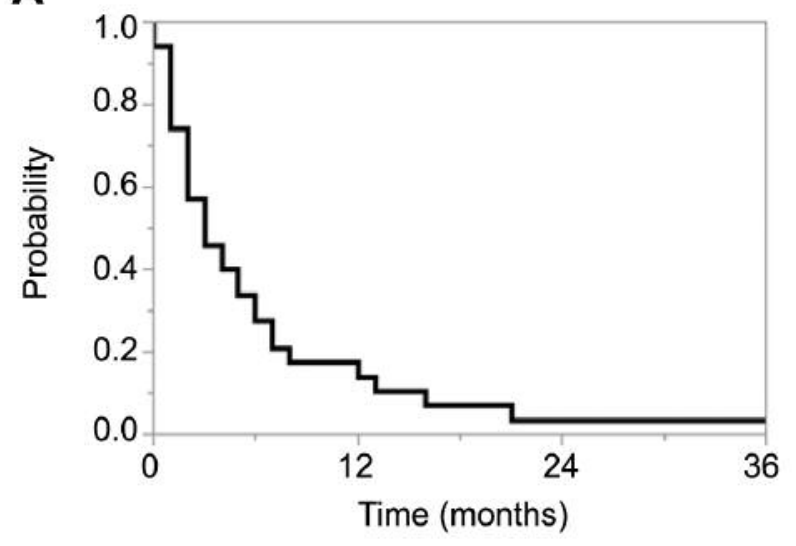

B

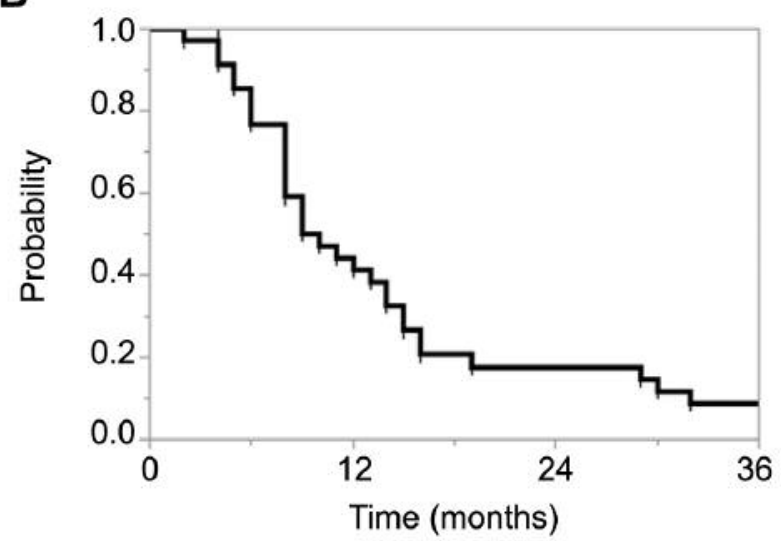

Figure 1. Kaplan-Meier curves of progression-free $(A)$ and overall $(B)$ survival in patients with recurrent glioma following salvage re-irradiation using CyberKnife.

\section{Results}

Patient characteristics. The patient characteristics are summarized in Table I. In terms of glioma grade, $17.1 \%$, $25.7 \%$, and $57.1 \%$ had grades 2,3 , and 4 (glioblastoma), respectively. Moreover, 28.6\%, 37.1\%, 8.6\%, 20.0\%, and $5.7 \%$ of the patients had performance status scores of 0,1 , 2,3 , and 4 , respectively, at the time of salvage re-irradiation. Patients routinely received concomitant temozolomide from 2006 onwards.

Toxicities. During the follow-up period, 4 patients $(11.4 \%)$ developed grade $\geq 2$ radiation-induced brain necrosis [2 patients $(5.7 \%)$ with grade 3 and 1 patient each $(2.9 \%)$ with grade 2 and 4 , respectively]. There were no other toxicities of grades $\geq 2$ among the patients.

The patient who developed grade 4 brain necrosis had been re-irradiated for two recurrent lesions using CyberKnife after conventional radiotherapy (66 Gy) for glioblastoma surrounding the right lateral ventricle. Brain necrosis with serous cyst formation occurred, and the cyst was resected 3 months later.

Local control and survival. All patients completed planned treatment without interruption. During the follow-up period, 32 patients $(91.4 \%)$ died of gliomas, two $(5.7 \%)$ survived, and one $(2.9 \%)$ was lost to follow-up. Twenty-nine patients $(82.9 \%)$ experienced local recurrences. The 1-year OS and PFS rates were $41.3 \%$ [95\% confidence interval $(\mathrm{CI})=26.2$ $58.2 \%$ ] and $13.9 \%$ (95\% CI=5.5-30.9\%), respectively (Figure 1). The median OS and PFS following re-irradiation were 9.0 months (95\% CI=6-16 months) and 3.0 months $(95 \% \mathrm{CI}=1-$ 6 months), respectively. When categorized by grade, the median OS was $12.0,8.0$, and 9.5 months for those with

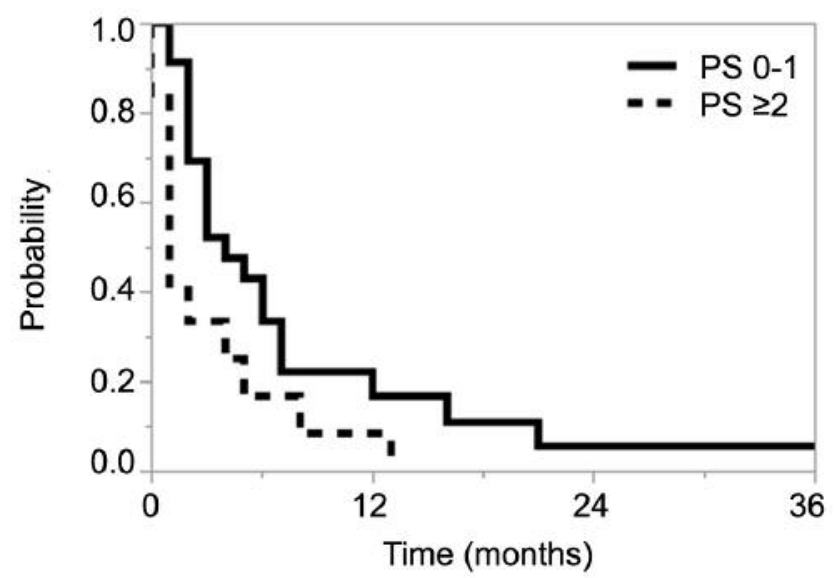

Figure 2. Kaplan-Meier curves of progression-free survival according to performance status $(P S)$ at the time of salvage re-irradiation of patients with recurrent glioma.

grade 2 gliomas, grade 3 gliomas, and glioblastomas, respectively.

Prognostic factors. Univariate analyses were performed to identify potential prognostic factors for PFS and OS among the different subgroups. Our results revealed that performance status at salvage re-irradiation was a significant predictor of PFS ( $p=0.034$ ) (Table II). Kaplan-Meier PFS plots according to the patient performance status at salvage re-irradiation are shown in Figure 2. The 1-year PFS rate of patients with performance status scores of $0-1$ at the time of re-irradiation was $16.7 \%$, whereas that of patients with scores of 2-4 was $8.3 \%$. No significant associations were observed in univariate analysis for OS. 
Table II. Univariate analysis of progression-free (PFS) survival and overall survival (OS).

\begin{tabular}{|c|c|c|c|c|c|}
\hline Factor & $\mathrm{N}$ & 1-Year PFS rate $(\%)$ & $p$-Value & 1 -Year OS rate $(\%)$ & $p$-Value \\
\hline \multicolumn{6}{|l|}{ Gender } \\
\hline Male & 24 & 9.1 & \multirow[t]{2}{*}{0.410} & 36.4 & \multirow[t]{2}{*}{0.545} \\
\hline Female & 11 & 16.2 & & 43.7 & \\
\hline \multicolumn{6}{|c|}{ Age at salvage re-irradiation } \\
\hline$\geq 57$ Years & 18 & 13.0 & \multirow[t]{2}{*}{0.706} & 41.3 & \multirow[t]{2}{*}{0.150} \\
\hline$<57$ Years & 17 & 14.7 & & 41.2 & \\
\hline \multicolumn{6}{|c|}{ Grading of the gliomas due to WHO classification } \\
\hline 2 & 6 & 0 & \multirow[t]{3}{*}{0.305} & 60 & \multirow[t]{3}{*}{0.297} \\
\hline 3 & 9 & 16.7 & & 33.3 & \\
\hline 4 & 20 & 15.0 & & 40.0 & \\
\hline \multicolumn{6}{|c|}{ PS score at salvage re-irradiation } \\
\hline $0-1$ & 23 & 16.7 & \multirow[t]{2}{*}{0.034} & 41.0 & \multirow[t]{2}{*}{0.211} \\
\hline$\geq 2$ & 12 & 8.3 & & 41.7 & \\
\hline \multicolumn{6}{|c|}{ Interval between initial irradiation and re-irradiation } \\
\hline$<11$ Months & 17 & 6.7 & \multirow{2}{*}{0.438} & 37.7 & \multirow[t]{2}{*}{0.359} \\
\hline$\geq 11$ Months & 18 & 20.8 & & 44.4 & \\
\hline \multicolumn{6}{|c|}{ Site of failure at salvage re-irradiation } \\
\hline Marginal & 2 & 0 & \multirow[t]{3}{*}{0.161} & 50.0 & \multirow[t]{3}{*}{0.697} \\
\hline In-field & 32 & 10.3 & & 38.8 & \\
\hline Out-of-field & 1 & 100 & & 100 & \\
\hline \multicolumn{6}{|c|}{ Salvage re-irradiation dose } \\
\hline$\geq 26 \mathrm{~Gy}$ & 17 & 19.6 & \multirow[t]{2}{*}{0.114} & 56.5 & \multirow[t]{2}{*}{0.628} \\
\hline$<26 \mathrm{~Gy}$ & 18 & 8.3 & & 27.8 & \\
\hline \multicolumn{6}{|c|}{ PTV at salvage re-irradiation } \\
\hline$\geq 4.2 \mathrm{ml}$ & 23 & 15.7 & \multirow[t]{2}{*}{0.951} & 34.8 & \multirow[t]{2}{*}{0.127} \\
\hline$<4.2 \mathrm{ml}$ & 12 & 11.1 & & 55.0 & \\
\hline \multicolumn{6}{|l|}{ Temozolomide } \\
\hline Yes & 6 & 16.7 & \multirow[t]{2}{*}{0.722} & 33.3 & \multirow[t]{2}{*}{0.787} \\
\hline No & 29 & 12.9 & & 43.0 & \\
\hline
\end{tabular}

N: Number of patients; WHO: World Health Organization; PS: performance status; PTV: planning target volume.

\section{Discussion}

Salvage radiotherapy via SRT using Linac or Gamma Knife has already been shown to be a reasonable treatment option for patients with gliomas who experience recurrences in the form of focal lesions following surgery and conventional radiotherapy (5-12). However, few studies to date have investigated the use of re-irradiation with CyberKnife for recurrent glioma $(11,12,14,15)$. Our findings demonstrated that re-irradiation with CyberKnife achieves moderate survival benefits with acceptable toxicity, and that performance status upon salvage re-irradiation is a significant predictor of PFS. Therefore, salvage re-irradiation using CyberKnife may be a viable option for patients with recurrent glioma, especially for those with good performance status scores.

Radiation-induced brain necrosis is generally considered a major toxicity risk after re-irradiation for recurrent glioma. Notably, however, there were no other toxicities of grade 2 or more among our patients. Several studies of SRS or SRT using Linac or Gamma Knife for recurrent gliomas revealed that grade 2 or more and grade 4 radiation-induced brain necrosis occurred in $6.4-29 \%$ and $3.8-5.5 \%$ of their treated patients, respectively $(5,8,17-20)$. Pinzi et al. reported that seven out of their 128 patients with high-grade gliomas who underwent re-irradiation using CyberKnife experienced radiation-induced necrosis, three of whom required surgical intervention; however, they did not report the grades of necrosis (12). Conti et al. reported that one $(4.3 \%)$ of their 23 patients with recurrent glioblastoma multiforme who underwent CyberKnife SRS experienced radiation-induced necrosis that required surgery (11). Levy et al. investigated survival rates for 13 patients who underwent CyberKnife reirradiation for recurrent glioma following chemoradiotherapy, and reported that three (23\%) developed radionecrosis without revealing their grades (15). In our present study, four (11.4\%) and one (2.9\%) of our patients who were re-irradiated with CyberKnife for recurrent glioma developed grade 2 or more and grade 4 radiationinduced brain necrosis, respectively. These toxicity rates are similar to those reported in patients who underwent Linac or Gamma Knife treatment.

Although several studies of patients with recurrent glioma who were treated with SRS or SRT re-irradiation have been 
performed using Linac or Gamma Knife, the median OS of these patients following re-irradiation was 5.3-13.5 months (5, 7-10, 17-21). Relatively fewer studies have investigated the use of re-irradiation with CyberKnife for recurrent glioma; these revealed a median post-re-irradiation OS of 7.0-14.0 months. $(11,12,14,15)$. The median OS among our patients following re-irradiation was 9.0 months. Such findings indicate that the survival benefits following reirradiation with CyberKnife are comparable to those following Linac or Gamma Knife treatment.

With respect to prognostic factors, studies that investigated patients with recurrent glioma re-irradiated using SRT or SRS $(12,14,22)$ found that the tumour histology at initial surgery, age, time between initial radiotherapy and re-irradiation, other therapies after reirradiation, and extent of surgical interventions were predictors of overall survival. Our results showed that performance status at salvage re-irradiation is a previously unreported significant predictor of PFS, although no significant predictors of OS were identified. Our finding is similar to that by Carson et al., who found that the Karnofsky performance status was a significant predictor of survival in patients with recurrent glioma who were treated with chemotherapy (23).

Our study had several limitations. Firstly, it was a singlecentre retrospective analysis using observational data. Secondly, the median follow-up period after re-irradiation was short (9.0 months) because patients with recurrent glioma have poor prognoses; consequently, our results might have underestimated the toxicity of re-irradiation with CyberKnife. Thirdly, as the sample size was small, it is premature to generalize our findings, especially in terms of prognostic factors. Finally, we included a wide variety of doses and fractionation schemes that may have influenced the treatment outcome; as such, additional prospective studies are warranted.

In conclusion, salvage re-irradiation using CyberKnife appears to be a feasible treatment option with an acceptable toxicity profile for patients with recurrent glioma, especially those with a good performance status.

\section{Conflicts of Interest}

The Authors declare that they have no competing interests.

\section{Authors' Contributions}

Kana Adachi: Study design, treatment of the patients, data assembly, statistical analyses and interpretation, writing of the article, revision of the article, and approval of the final article. Kazuhiko Hayashi: Study design, data assembly, statistical analyses and interpretation, writing of the article, revision of the article, and approval of the final article. Naoki Kagawa: Study design, treatment of the patients, data assembly, and approval of the final article. Manabu Kinoshita:
Study design, treatment of the patients, data assembly, and approval of the final article. Iori Sumida: Study design, data interpretation, and approval of the final article. Yuichi Akino: Data interpretation, revision of the article, and approval of the final article. Hiroya Shiomi: Study design, treatment of the patients, and approval of the final article. Keisuke Tamari: Study design, data interpretation, writing of the article, and approval of the final article. Osamu Suzuki: Study design, data interpretation, writing of the article, and approval of the final article. Ryuichi Hirayama: Data interpretation, and approval of the final article. Noriyuki Kijima: Data interpretation, and approval of the final article. Fumiaki Isohashi: Data interpretation, and approval of the final article. Yuji Seo: Data interpretation, and approval of the final article. Keisuke Otani: Study design, data assembly, statistical analyses and interpretation, and approval of the final article. Haruhiko Kishima: Study design, data interpretation, revision of the article, and approval of the final article. Kazuhiko Ogawa: Study design, data interpretation, revision of the article, and approval of the final article.

\section{References}

1 Rasmussen BK, Hansen S, Laursen RJ, Kosteljanetz M, Schultz H, Nørgård BM, Guldberg R and Gradel KO: Epidemiology of glioma: clinical characteristics, symptoms, and predictors of glioma patients grade I-IV in the Danish Neuro-Oncology Registry. J Neurooncol 135(3): 571-579, 2017. PMID: 28861666. DOI: $10.1007 / \mathrm{s} 11060-017-2607-5$

2 Wesseling P and Capper D: WHO 2016 Classification of gliomas. Neuropathol Appl Neurobiol 44(2): 139-150, 2018. PMID: 28815663. DOI: 10.1111/nan.12432

3 Rapp M, Baernreuther J, Turowski B, Steiger H-J, Sabel M and Kamp MA: Recurrence pattern analysis of primary glioblastoma. World Neurosurg 103: 733-740, 2017. PMID: 28434963. DOI: 10.1016/j.wneu.2017.04.053

4 Birk HS, Han SJ and Butowski NA: Treatment options for recurrent high-grade gliomas. CNS Oncol 6(1): 61-70, 2017. PMID: 28001091 DOI: 10.2217/cns-2016-0013

5 Patel M, Siddiqui F, Jin JY, Mikkelsen T, Rosenblum M, Movsas B and Ryu S: Salvage reirradiation for recurrent glioblastoma with radiosurgery: Radiographic response and improved survival. J Neurooncol 92(2): 185-191, 2009. PMID: 19066727. DOI: $10.1007 / \mathrm{s} 11060-008-9752-9$

6 Fogh SE, Andrews DW, Glass J, Curran W, Glass C, Champ C, Evans JJ, Hyslop T, Pequignot E, Downes B, Comber E, Maltenfort M, Dicker AP and Werner-Wasik M: Hypofractionated stereotactic radiation therapy: An effective therapy for recurrent high-grade gliomas. J Clin Oncol 28(18): 3048-3053, 2010. PMID: 20479391. DOI: 10.1200/JCO. 2009.25.6941

7 Fokas E, Wacker U, Gross MW, Henzel M, Encheva E and Engenhart-Cabillic R: Hypofractionated stereotactic reirradiation of recurrent glioblastomas: A beneficial treatment option after high-dose radiotherapy? Strahlenther Onkol 185(4): 235-240, 2009. PMID: 19370426. DOI: 10.1007/s00066-009-1753-x

8 Henke G, Paulsen F, Steinbach JP, Ganswindt U, Isijanov H, Kortmann RD, Bamberg $\mathrm{M}$ and Belka C: Hypofractionated reirradiation for recurrent malignant glioma. Strahlenther Onkol 185(2): 113-119, 2009. PMID: 19240998. DOI: 10.1007/s00066009-1969-9

9 Skeie BS, Enger PØ, Brøgger J, Ganz JC, Thorsen F, Heggdal $\mathrm{JI}$ and Pedersen P-H: $\gamma$ Knife surgery versus reoperation for 
recurrent glioblastoma multiforme. World Neurosurg 78(6): 658 669, 2012. PMID: 22484078. DOI: 10.1016/j.wneu.2012.03.024

10 Martínez-Carrillo M, Tovar-Martín I, Zurita-Herrera M, Del Moral-Ávila R, Guerrero-Tejada R, Saura-Rojas E, OsorioCeballos JL, Arrebola-Moreno JP and Expósito-Hernández J: Salvage radiosurgery for selected patients with recurrent malignant gliomas. Biomed Res Int 2014: 657953, 2014. PMID: 24895599. DOI: $10.1155 / 2014 / 657953$

11 Conti A, Pontoriero A, Arpa D, Siragusa C, Tomasello C, Romanelli P, Cardali S, Granata F, De Renzis C and Tomasello F: Efficacy and toxicity of CyberKnife re-irradiation and "dose dense" temozolomide for recurrent gliomas. Acta Neurochir 154(2): 203-209, 2012. PMID: 21984132. DOI: 10.1007/s00701011-1184-1

12 Pinzi V, Orsi C, Marchetti M, Milanesi IM, Bianchi LC, DiMeco F, Cuccarini V, Farinotti M, Ferroli P, Finocchiaro G, Franzini A, Fumagalli M, Silvani A and Fariselli L: Radiosurgery reirradiation for high-grade glioma recurrence: A retrospective analysis. Neurol Sci 36(8): 1431-1440, 2015. PMID: 25805705. DOI: $10.1007 / \mathrm{s} 10072-015-2172-7$

13 Chang SD and Adler JR: Robotics and radiosurgery - The Cyberknife. Stereotact Funct Neurosurg 76(3-4): 204-208, 2001. PMID: 12378098. DOI: 10.1159/000066719

14 Villavicencio AT, Burneikiene S, Romanelli P, Fariselli L, McNeely L, Lipani JD, Chang SD, Nelson EL, McIntyre M, Broggi $G$ and Adler JR: Survival following stereotactic radiosurgery for newly diagnosed and recurrent glioblastoma multiforme: A multicenter experience. Neurosurg Rev 32(4): 417-424, 2009. PMID: 19633875. DOI: 10.1007/s10143-0090212-6

15 Levy S, Chapet S, Scher N, Debbi K, Ruffer A, Bernadou G, Pointreau Y, and Calais G: Reirradiation of gliomas under stereotactic conditions: Prognostic factors for survival without relapse or side effects, a retrospective study at Tours regional University Hospital (France). Cancer Radiother 21(8): 759-765, 2017. PMID: 29128197. DOI: 10.1016/j.canrad.2017.05.006

16 Common Terminology Criteria for Adverse Events version 4.0. U.S. Department of Health and Human Services, National Institutes of Health, National Cancer Institute, 2009. Available at: https://www.eortc.be/services/doc/ctc/CTCAE_4.03_2010-0614_QuickReference_5x7.pdf. Last accessed 15th March 2019.
17 Maranzano E, Anselmo P, Casale M, Trippa F, Carletti S, Principi $\mathrm{M}$, Loreti $\mathrm{F}$, Italiani $\mathrm{M}$, Caserta $\mathrm{C}$ and Giorgi $\mathrm{C}$ : Treatment of recurrent glioblastoma with stereotactic radiotherapy: Long-term results of a mono-institutional trial. Tumori 97(1): 56-61, 2017. PMID: 21528665.

18 Koga T, Maruyama K, Tanaka M, Ino Y, Saito N, Nakagawa K, Shibahara $\mathrm{J}$ and Todo T: Extended field stereotactic radiosurgery for recurrent glioblastoma. Cancer 118(17): 4193-4200, 2012. PMID: 22180028. DOI: $10.1002 / \mathrm{cncr} .27372$

19 Biswas T, Okunieff P, Schell MC, Smudzin T, Pilcher WH, Bakos RS, Vates GE, Walter KA, Wensel A, Korones DN and Milano MT: Stereotactic radiosurgery for glioblastoma: retrospective analysis. Radiat Oncol 4: 11, 2009. PMID: 19292912. DOI: $10.1186 / 1748-717 X-4-11$

20 Elliott RE, Parker EC, Rush SC, Kalhorn SP, Moshel YA, Narayana A, Donahue B and Golfinos JG: Efficacy of gamma knife radiosurgery for small-volume recurrent malignant gliomas after initial radical resection. World Neurosurg 76(1-2): 128-140; discussion 61-62, 2011. PMID: 21839964. DOI: 10.1016/ j.wneu.2010.12.053

21 Anand AK, Kumar P, Patir R, Vaishya S, Bansal AK, Chaudhoory AR, Punnakal AU, Malhotra $\mathrm{H}$ and Munjal RK: Fractionated stereotactic radiosurgery with volumetric modulated arc therapy (Rapid Arc) for reradiation in recurrent high-grade gliomas. J Cancer Res Ther 10(1): 97-102, 2014. PMID: 24762494. DOI: 10.4103/0973-1482.131403

22 Combs SE, Edler L, Rausch R, Welzel T, Wick W and Debus J: Generation and validation of a prognostic score to predict outcome after re-irradiation of recurrent glioma. Acta Oncol 52(1): 147-152, 2013. PMID: 22686472. DOI: 10.3109/ 0284186X.2012.692882

23 Carson KA, Grossman SA, Fisher JD and Shaw EG: Prognostic factors for survival in adult patients with recurrent glioma enrolled onto the New Approaches to Brain Tumor Therapy CNS Consortium phase I and II clinical trials. J Clin Oncol 25(18): 26012606, 2007. PMID: 17577040. DOI: 10.1200/ JCO.2006.08.1661

Received April 29, 2019

Revised May 12, 2019

Accepted May 17, 2019 